\title{
The comparative study of three nonparametric methods of SAR tomography for building reconstruction
}

\author{
Mehrnoosh Omati ${ }^{\mathrm{a}}$, Mahmod Reza Sahebi ${ }^{\mathrm{a}, *}$, Hossein Aghababaei ${ }^{\mathrm{b}}$ \\ ${ }^{a}$ Faculty of Geodesy and Geomatics Engineering, Remote Sensing Institute, K. N. Toosi University of Technology, Tehran 19967-15433, Iran \\ ${ }^{\mathrm{b}}$ Faculty of Geo-Information Science and Earth Observation (ITC), University of Twente, 7514AE Enschede, the Netherlands
}

Received 14 March 2021; received in revised form 3 July 2021; accepted 31 July 2021

\begin{abstract}
Over the past years, synthetic aperture radar (SAR) tomography (TomoSAR) has indicated significant potentials for threedimensional (3D) reconstruction of buildings in urban areas. A large number of SAR images are thus required to perform tomographic inversions in non-parametric spectral estimators. To this end, in the case of a limited choice of SAR acquisitions, the present study aims to evaluate the capabilities of five non-parametric spectral estimation (SE) techniques, including linear prediction (LP), maximum entropy (ME), and minimum norm (MN), Capon, and beamforming (BF) in the tomographic reconstruction of urban environments. The performance analysis is carried out by using both simulated and real SAR datasets. The study results demonstrate that the proposed efficient LP estimator, minimizing the average output signal power over the antenna array elements, can separate the layover scatterers along the height direction. This low-computational SE method is thus able to clean side lobes while using a small number of observations. The proposed algorithms, as applied on TerraSAR-X strip-map images of the city of Tehran, Iran, verify the effectiveness of the non-parametric LP reconstruction technique for urban buildings. The estimated height of the scatterers also indicates that the LP estimator is similar to the field-based measurements once compared with ME, MN, Capon, and BF reconstruction methods.
\end{abstract}

(C) 2021 COSPAR. Published by Elsevier B.V. All rights reserved.

Keywords: Synthetic aperture radar tomography (TomoSAR); Nonparametric spectral estimation methods; LINEAR prediction (LP); Maximum entropy (ME); Minimum norm (MN); Urban building reconstruction

\section{Introduction}

Nowadays, high spatial concentration of scatterers within a range-azimuth resolution cell due to the interference of buildings, houses, roads, bridges, trees, and vegetation along with analysis of synthetic aperture radar (SAR) data in urban areas has turned into an active field of research (Guillaso et al., 2014). Interferometric SAR (InSAR), as a remote sensing technique, can exploit the phase difference of coherent radar signals from slightly dif-

\footnotetext{
* Corresponding author.

E-mail addresses: momati@mail.kntu.ac.ir (M. Omati), sahebi@kntu. ac.ir (M.R. Sahebi), h.aghababaei@utwente.nl (H. Aghababaei).
}

ferent positions, to generate a three-dimensional (3D) ground surface reconstruction (Fornaro, 2014).

Given the side-looking mode of the SAR system, geometric distortions, including foreshortening and layover might frequently occur in urban areas (Stilla et al., 2003). In the presence of steep topography, such as vertical structures (i.e., buildings), the superposition of multiple scattering contributions may accordingly take place in SAR image pixels (Lombardini et al., 2013). Besides, the difficulty of the phase unwrapping operation at the edges of buildings and other corner-reflectors in imaged scenes can make the InSAR processing performance less effective (Schmitt and Stilla, 2014). 
To deal with these limitations, SAR tomography (TomoSAR), as a multi-baseline technique of InSAR configuration, has recently received significant attention in the case of buildings in urban environments. This modern method employs a stack of SAR images collected at slightly different flight tracks to reconstruct the height distribution of backscattered power (Gini et al., 2002).

A wide variety of spectral estimation (SE) methods can be thus applied to resolve the inverse problem of the TomoSAR imaging technique (Stoica and Moses, 2005). SE algorithms with applications to TomoSAR can be accordingly grouped into two main categories: singlelooking and multi-looking methods (Zhu, 2011). In this sense, single-looking algorithms utilize a complex-valued image stack of single SAR image azimuth-range pixels and do not care about interrelationships of adjacent pixels. For multi-looking algorithms, SAR data can be represented in a complex covariance matrix form by averaging backscattered signals of surrounding pixels. In general, by taking multiple looks of SAR measurements, multi-look processing enhances signal-to-noise ratio (SNR) in an elevation direction (Zhu, 2011). Accordingly, different SE techniques have been thus far implemented, including conventional beamforming (BF), singular value decomposition (SVD), Capon, and multiple signal classification (MUSIC) (Fornaro et al., 2003; Lombardini and Reigber, 2003; Stoica and Moses, 1997; Gini and Lombardini, 2005).

Within another categorization, various SE methods can be also grouped under three heads: sparsity-based compressive sensing (CS) (Zhu and Bamler, 2010), together with parametric and non-parametric techniques (Gini et al., 2002). With regard to the iterative procedure and the unavailability of adaptive convex optimization algorithms, the main drawback of CS-based TomoSAR imaging techniques is associated with high computational complexity (Martin Del Campo et al., 2018). Besides, the parametric SEs are based on prior knowledge about the statistical model of received signals and the number of interfering scatterers in an azimuth-range image pixel. Commonly, parametric estimators may represent better estimation if the data appear to agree closely with the assumed model (Wei et al., 2014).

Compared with the first two groups, for non-parametric SE methods, no assumption about distributional model parameters within a resolution cell is required. The number of scatterers and their unknown parameters can be thus directly estimated from the SAR image data (Wei et al., 2014). Therefore, the non-parametric SEs become more applicable to image processing. However, some nonparametric estimators such as beamforming and Capon might achieve low height resolution while having high sidelobe levels. Generally, the non-parametric SEs can work well if a large number of acquisitions are available. One problem to be deliberated is that the quality of nonparametric reconstruction methods is lost once the number of image data diminishes.
Primarily, this paper aims to investigate the quality of TomoSAR reconstruction and to reflect on the accuracy of building height retrieval, using a limited number of acquisitions available. To this end, three direction-ofarrival (DOA) estimation techniques, i.e., linear prediction (LP), maximum entropy (ME), and minimum norm (MN) with the TomoSAR application in urban areas are introduced. The evaluation of these new non-parametric SEs is also conducted in two perspectives: the performance of each method in the separation of multiple scatterers inside an azimuth - range resolution cell, as well as the robustness of the non-parametric SEs, applied to the reduced number of images. Interestingly, the LP-based SE method minimizing the average output signal power over the antenna array elements accounts for the potentialities of TomoSAR processing to a dataset of TerraSAR-X stripmap data. The proposed LP estimator is also endowed with a strong capability to discriminate layover scatterers located at different elevation coordinates, suppress the sidelobe effect, and retain TomoSAR reconstruction quality with respect to the reduced number of SAR images.

\section{Methodology}

The implementation workflow of the proposed nonparametric SE techniques of TomoSAR on TerraSAR-X stripmap acquisitions is demonstrated in Fig. 1. Upon fulfilling the necessary preprocessing steps, three novel DOA estimation methods with applications to TomoSAR are applied over the urban area. The proposed estimator techniques, namely, LP, ME, and MN, are conducted to evaluate the performance quality of the employed methods in layover separation ability, the sidelobe level reduction in the tomography reconstruction, the robustness against a limited number of SAR acquisitions, and the height retrieval accuracy of the building case study.

\subsection{TomoSAR imaging technique}

The scheme of the TomoSAR imaging geometry for urban areas is shown in Fig. 2. Accordingly, the backscattered signals of the scatterers, located at different heights, may interfere inside the same SAR resolution cell in the side-looking geometry of the SAR acquisition. For complex-built geometry, the TomoSAR technique is capable to discriminate multiple scatterers in each pixel through the formation of the synthetic aperture principle along the elevation direction.

In a stack of Nco-registered complex-valued SAR images, the received signal of the azimuth-range pixel $(x, r)$ at the $n^{\text {th }}$ image from all the scatterers can be expressed in the form Eq. (1) (Fornaro et al., 2003):

$$
\begin{aligned}
& g_{n}(x, r)=\int \gamma(s) \exp \left[-j 2 \pi \xi_{n} s\right] d s+\varepsilon_{n}(x, r) \\
& g=A \cdot \gamma+\varepsilon
\end{aligned}
$$




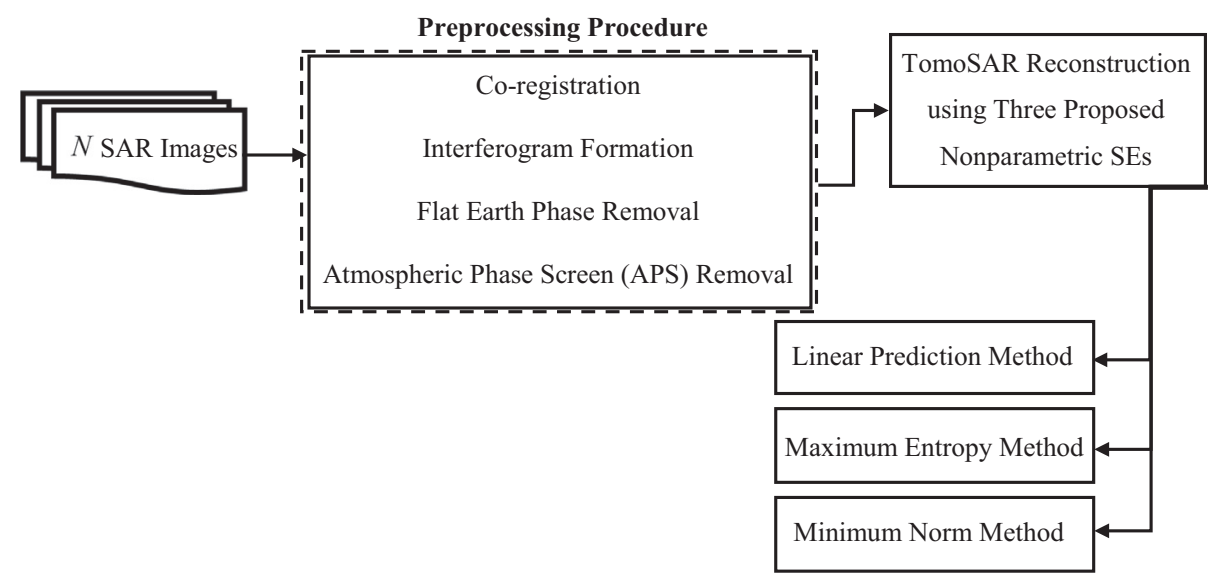

Fig. 1. Proposed SAR tomographic processing framework.

where $\xi_{n}=\frac{2 b_{\perp n}}{\lambda r}$ is spatial frequency along the elevation direction, the parameter $\lambda$ represents wavelength, $r$ is the measured distance between the radar antenna and target, $b_{\perp n}$ is the perpendicular baseline component between each slave image and master image, and $\gamma($.) is the complexvalued unknown reflectivity vector along the elevation direction for each azimuth-range pixel. The noise term $\varepsilon_{n}$ can be neglected if appropriate pre-processing of the SAR image stack is performed.

Through uniformly sampling the continuous reflectivity function along elevation direction with $L$ steps, the steering matrix $A=\left[a_{1}, a_{2}, \cdots, a_{L}\right]$ is composed of $N L$-dimensional steering vectors as columns. The $N$ dimensional steering vector $a_{l}$ associated with each scatterer in elevation dimension can be written in the form Eq. (2):

$a_{l}=\left[e^{\left(j 2 \pi \xi_{1} s_{l}\right)}, e^{\left(j 2 \pi \xi_{2} s_{l}\right)}, \cdots, e^{\left(j 2 \pi \xi_{N} s_{l}\right)}\right]^{T}(l=1,2, \cdots, L)$

where $s_{l}(l=1,2, \cdots, L)$ represents the $l^{\text {th }}$ discrete elevation position and $\gamma\left(s_{l}\right)$ is the reflectivity power of the scatterer at the elevation position $s_{l}$. The spectral estimation methods with application to SAR tomography promise to retrieve the vertical distribution of the backscattered power for

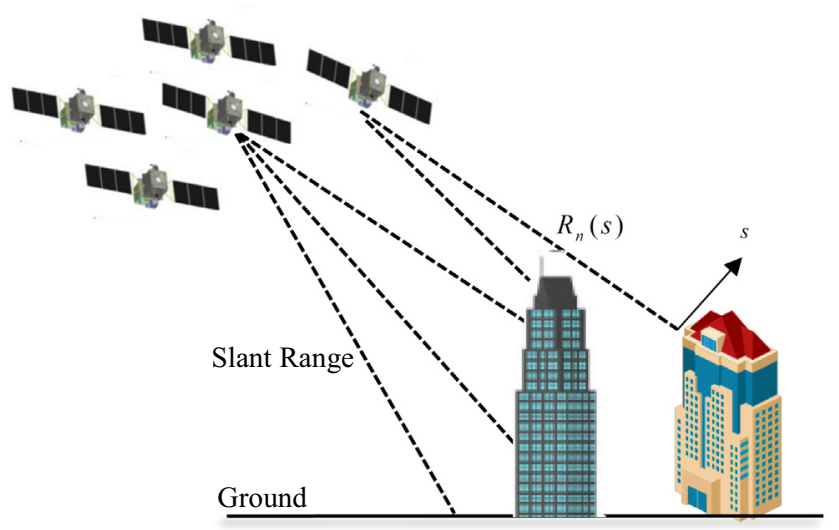

Fig. 2. TomoSARacquisition geometry in urban. each azimuth-range pixel. In the following, we introduce three non-parametric spectral estimators for this purpose.

\subsection{DOA estimation techniques in SAR tomography}

Based on the multiple baseline SAR observations over the same target, the obtained complex data can be considered as the received signals by an array of antenna elements (Kato et al., 2015). Thus, tomographic imaging can be performed by DOA-based estimation methods after the preprocessing of SAR images, including co-registration, phase flattening, and atmospheric phase screen (APS) removal. This study introduces three new non-parametric DOA algorithms for performance evaluation of the proposed estimators with respect to accurate height reconstruction of buildings and robustness to the reduced number of images in 3D SAR imaging.

\subsubsection{Linear prediction DOA estimator}

The linear prediction method is a potentially valuable tool in a wide range of applications such as time-series problems, speech signal analysis, array signal processing, and spectrum analysis (Makhoul, 1975). The basic concept of the proposed LP technique for power spectrum estimation is the minimization of the average output signal power over the array of antenna elements (Gamba, 2020; Johnson, 1982). Under this constraint, the LP as a noiserobust non-parametric SE makes it possible to retrieve the building height from the stack of SAR images.

The estimation of the value of $g_{n}$ in the LP estimator method is based on a weighted linear combination of the $\exp \left[-j 2 \pi \xi_{n} s_{l}\right]$ with predictive coefficients defined from $\gamma\left(s_{l}\right)$. The formulation for LP estimator can be written as Eq. (3):

$$
\begin{aligned}
g_{n} & =\sum_{l=1}^{L} \gamma\left(s_{l}\right) \exp \left[-j 2 \pi \xi_{n} s_{l}\right]+\varepsilon_{n} \quad n=1,2, \cdots, N l \\
& =1,2, \cdots, L
\end{aligned}
$$


The basic idea of the nonparametric linear prediction estimation method is the finding of the column vector of weights $\gamma(s)=\left[\gamma\left(s_{1}\right), \gamma\left(s_{2}\right), \cdots, \gamma\left(s_{L}\right)\right]^{T}$ for each row in the steering matrix to minimize the following criterion in the form Eq. (4):

$E\left\{\left|\gamma(s)^{H} g\right|^{2}\right\}=E\left\{\gamma(s)^{H} g g^{H} \gamma(s)\right\}=\gamma(s)^{H} C_{g} \gamma(s)$

The optimal solution of the constrained problem can be obtained from minimizing the quantity of the objective criterion subject to a unity-based weight vector on the selected elevation position. The constraint can be defined in the form Eq. (5) (Johnson, 1982):

$\gamma(s)^{H} u=1$

The $i^{\text {th }}$ column of an identity matrix $I_{N \times N}$ is the column vector $u$. In the linear prediction method as a multilookingbased spectral estimator, the estimation of the covariance matrix elements is obtained by spatial averaging neighboring image pixels in both principal SAR imaging directions. The estimated covariance matrix can be calculated as Eq. (6):

$C_{g}=E\left\{g g^{H}\right\} \approx C_{g}=\frac{1}{M} \sum_{m=1}^{M} g g^{H}$

where $(\cdot)^{\mathrm{H}}$ and $E[\cdot]$ are, respectively, the Hermitian and Expectation operators. The $M$ refers to the total number of pixels in the neighborhood that need to be averaged. In the following, the Lagrange multiplier approach is used to solve the optimal weight vector of the LP estimator. The resulting best vector can be represented in the form of Eq. (7) (Gamba, 2020; Johnson, 1982):

$\gamma_{\text {opt }}(s)=\frac{C_{g}^{-1} u}{u^{H} C_{g}^{-1} u}$

In the linear prediction method corresponding to the autoregressive (AR) model, the estimated power spectrum is obtained based on the mean squared error of the prediction divided by the magnitude squared spectrum of the prediction weights. Thus, the estimated power spectrum can be defined as Eq. (8) (Gamba, 2020; Johnson, 1982):

$P_{L P}(s)=\frac{u^{H} C_{g}^{-1} u}{\left|u^{H} C_{g}^{-1} A\right|^{2}}$

As expressed in Eq. (8), the choice of column $u$ affects the performance of the LP estimator. Therefore, the maximum contrast of the reflectivity profile criterion is proposed to achieve the most effective possible column. The idea behind the selected vector, $u$, is the reconstructed reflectivity profile with maximum contrast among all possible profiles extracted from the identity matrix columns.

\subsubsection{Minimum norm DOA estimator}

The subspace-based minimum norm method is known for its high-resolution capability in the DOA estimation (Ermolaev and Gershman, 1994). In this technique, after the covariance matrix estimation $C_{g}$, the singular value decomposition (SVD) method is applied to decompose it into the product of three following matrices $C_{g}=U S V^{T}$. The columns of the noise subspace are formed by selecting the subset of the columns of eigenvectors from $U$, such that $U_{\text {Noise }}=U(:, q+1: N)$. The parameter of $q$ refers to the number of the largest eigenvalues of the estimated covariance matrix.

The minimum norm method aims to identify the optimal weight vector $d=\left[d_{1}, d_{2}, \cdots, d_{N}\right]^{T}$, that is, the weight vector can be expressed as a linear combination of noise eigenvectors. The solution to the optimization problem can be obtained by the Eq. (9):

$\min d^{H} d, U_{\text {Signal }}^{H} d=0, d^{H} e_{1}=1$

where $e_{1}$ is the first column vector from an $N \times N$ identity matrix (the vector whose first element is 1 and 0 elsewhere). $U_{\text {Signal }}=\left[u_{1}, u_{2}, \cdots, u_{q}\right]$ as a signal subspace matrix of the estimated covariance matrix is derived from the eigenvectors corresponding to the $q$ largest eigenvalues. In this method, the weight vector is constrained to lie in the noise subspace and to provide a resulting vector of $d$ with the minimum norm. A column vector containing zeros in all elements except one in the first row satisfies this given condition. The solution of the optimization problem in Eq. (9) will yield the power spectrum of the MN method. This estimator can be written as Eq. (10):

$P_{M N}(s)=\frac{1}{\left|A^{T} U_{\text {Noise }} U_{\text {Noise }}^{H} e_{1}\right|^{2}}$

\subsubsection{Maximum entropy DOA estimator}

In (Makhoul, 1975; Gamba, 2020) it is shown that the maximum entropy estimator is equivalent to an autoregressive (AR) model, fitting the data by the method of least squares. The covariance matrix extrapolation is also assumed as the basis of the ME DOA estimation technique (Makhoul, 1975). The extrapolation is thus carried out with the aim of signal entropy maximization, performed by searching autoregressive (namely, prediction) coefficients (Gamba, 2020). The coefficients of the optimization problem can be obtained by minimizing the expected prediction error subject to the condition $\omega e_{1}$, where $\omega=\left[\omega_{1}, \omega_{2}, \cdots, \omega_{N}\right]$ is the vector of AR coefficients and $e_{1}=[1,0, \cdots, 0]$ is the first column of the identity matrix. In this method, using the Lagrange multiplier method, the AR coefficients can be defined as Eq. (11):

$\omega=\frac{C_{g}^{-1} e_{1}}{e_{1}^{T} C_{g}^{-1} e_{1}}$

The output maximum entropy power spectrum is given by Eq. (12):

$P_{M E}(s)=\frac{1}{\left|A^{T} C_{j}\right|^{2}}$ 


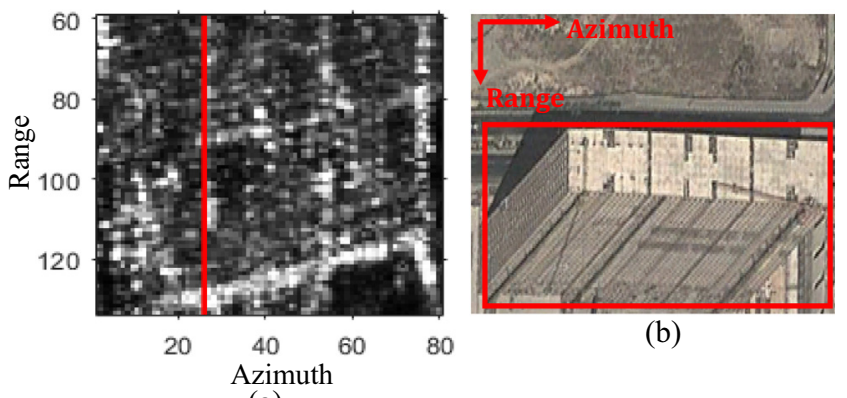

(a)

Fig. 3. Case study over Third Millennium Tower. (a) The mean amplitude SAR image. (b) the location of the Tower on Google Earth.

where $C_{j}$ is the $j^{\text {th }}$ column vector of the inverse of the covariance matrix. The choice of column $C_{j}$ has a significant effect on the performance of the maximum entropy spectrum technique. In a similar way, the maximum contrast criterion is adapted to select the optimum column of the matrix.

\section{Study area and dataset}

In this survey, the study area is the Third Millennium Tower, covering a region with $74 \times 81$ pixels in the image (Fig. 3). The tower, centered at $35^{\circ} 44^{\prime} 53.001^{\prime \prime} \mathrm{N}, 51^{\circ} 23^{\prime}$ $50.528^{\prime \prime} \mathrm{E}$ with a height of $120 \mathrm{~m}$, is known as one of the tallest buildings in Iran. Fig. 3 shows the mean amplitude of the SAR image and the location of the Third Millennium Tower on Google Earth.

For this research, a stack of 19 stripmap images is acquired by the TerraSAR-X sensor, allowing the performance assessment of the proposed TomoSAR methods. The SAR images were taken over the city of Tehran, Iran, from descending orbit direction between 2012 and 2013. The polarization mode of the images is $\mathrm{HH}$, and the values of the slant range and azimuth resolutions are $1.2 \mathrm{~m}$ and $3.3 \mathrm{~m}$, respectively. The total spatial baseline span of distribution for the used images is about $414 \mathrm{~m}$, thus, the Rayleigh resolution criterion along the vertical direction is about $21 \mathrm{~m}$. The parameters of the TerraSAR-X SAR sensors are summarized in Table 1.

\section{Experiments and results}

\subsection{Numerical experiments}

In this simulated stack of SAR data, to investigate the robustness of the estimators to reduce the number of SAR images, the layover scatterers separation and elevation estimation is evaluated with three different values of the number of image acquisitions $N=10, N=22$, and $N=57$. In all scenarios, the total baseline is fixed. Fig. 4 shows the results of the reconstructed reflectivity profiles using five DOA estimation methods. The results obtained from the ME, MN, Capon, and BF estimators on simulated data affirm that two discrete scatterers inside per pixel can be identified and separated if the number of observations is increased. The findings indicate that the reliable elevation estimation in $\mathrm{ME}, \mathrm{MN}$, Capon, and $\mathrm{BF}$ estimators depends on the number of used images. Whiles, the results confirm that the proposed LP technique can detect two interfering scatterers located in a pixel with a reduced number of observations. This method demonstrates robustness to the number of acquisitions when the total baseline is kept constant.

\subsection{Results of proposed TomoSAR imaging algorithms on real dataset}

As depicted in Fig. 3(a), the top section of the building from the case study is at the near range and the bottom appears at the far range in the SAR images due to the effects of the SAR imaging geometry in the target interaction. The height difference estimations are conducted along the specified slice marked by the red line.

In the urban environment, at the intersection of the building facade facing the SAR sensor and the ground, more than one scatterer is mainly located at the same SAR image pixel. The TomoSAR imaging technique quality can be expressed by reducing noise levels in TomoSAR reconstruction, robustness to the reduced number of acquisitions, and height estimation accuracy, i.e., no difference between estimated height and ground-truth value. Fig. 5 shows the reconstructed reflectivity profiles with reference to the LP, ME, MN, Capon, and BF methods in the height-range plane.

The experimental results of the LP-based SE method for the Third Millennium Tower indicate the height difference between the pixel on the top and the one at the bottom section is $120 \mathrm{~m}$. This estimated value is the same as the actual height of the building. In the middle of the tomogram, it is observed that the LP estimator can resolve the layover problem by separating the overlaid scatterers within a pixel. Moreover, this method benefits from the reduction of the noise level in the reconstructed height-reflectivity profile.

The study results imply that the other employed TomoSAR methods, namely, ME, MN, Capon, and BF, suffer from the drawbacks of serious layover problems and poor sidelobe interference reduction. These methods denote strong height discontinuities in the reconstructed tomo-

Table 1

Terrasar-X Parameters.

\begin{tabular}{|c|c|c|c|c|}
\hline Wavelength (m) & Range $(\mathrm{km})$ & Incidence Angle & Slant Range Resolution (m) & Azimuth Resolution (m) \\
\hline 0.031 & 563 & $25^{\circ}$ & 1.2 & 3.3 \\
\hline
\end{tabular}


graphic profiles. The results obtained from the $\mathrm{ME}, \mathrm{MN}$, Capon, and BF estimators, affirm that these are highly affected by the improper baseline distribution, while LP shows more robust tomographic reconstruction. There are also considerable differences in the building height between the estimated value and the ground-truth data. The height estimations obtained from different spectral methods and their differences with the ground-truth are outlined in Table 2.

\subsection{Robustness of the DOA estimation techniques to the reduced number of $S A R$ images}

To achieve a reliable TomoSAR reconstruction, the performance of the non-parametric SE techniques for the reduction of the required number of baseline acquisitions need to be studied. To this end, a stack of data containing half of the SAR acquisitions is employed (i.e., a dataset comprised of ten images). The reduction of the number of passes is performed such that the overall baseline distribution of the ten SAR images is similar to the one with the number of all images. Fig. 6 shows the results of the five SEs in the building case study. Comparing the LP-based SE and the four other estimators proves the robustness of the proposed LP technique in minimizing the number of SAR images for TomoSAR reconstruction. The LP- based SE method illustrates a much better resistance against the sidelobe effect in the reconstructed reflectivity profiles compared with the $\mathrm{ME}, \mathrm{MN}$, Capon, and BF techniques, whereas these techniques are highly impaired in the case of the reduced number of distributed revisits. Compared with the reference height of the building, there are significant height differences obtained from the ME, MN, Capon, and BF estimators. Table 3 presents the results of the employed non-parametric SE methods using the limited number of acquisitions for the subset area examined in this study.

\subsection{Selecting the best column for linear prediction and maximum entropy estimators}

As mentioned, selecting a specific column vector from the identity matrix and the inverse of the covariance matrix can have considerable impacts on the results of the LP and ME DOA estimation methods, respectively. This part of the research study aims at proposing a new approach for selecting the optimal column of the $N \times N$ identity matrix and the inverse of the covariance matrix, using the contrast criterion. In the proposed method, first, the matrix of the size $N \times L$ consists of $N L$-dimensional vertical reflectivity power vectors is formed at each pixel of the SAR image. For the LP and ME estimators, each column of the

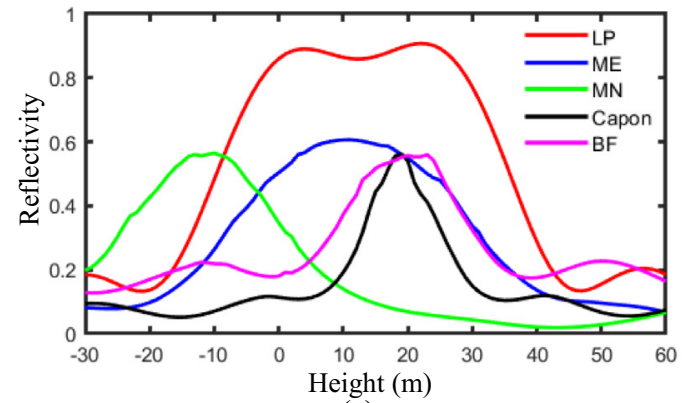

(a)

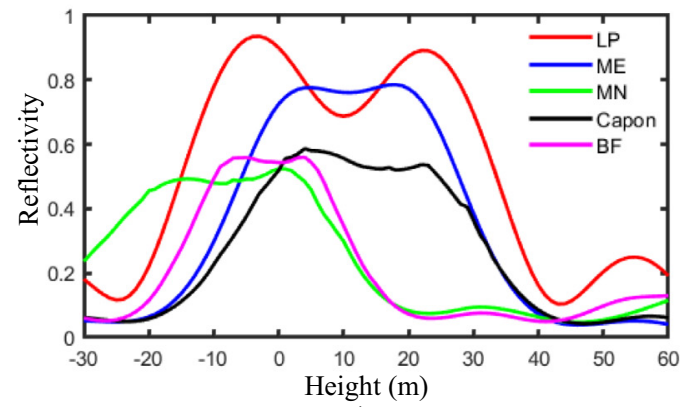

(b)

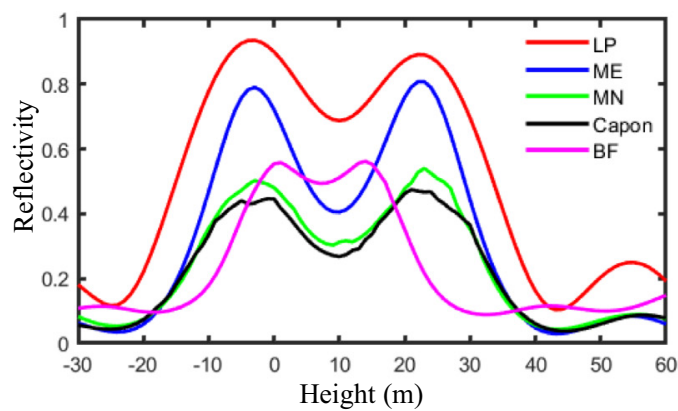

(c)

Fig. 4. Results of the reflectivity profile on the simulated dataset. (a) With 10 looks, (b) With 22 looks, (c) With 57 looks.

Table 2

Estimated building heights and their differences for third millennium tower.

\begin{tabular}{llllll}
\hline DOA Method & Linear Prediction & Maximum Entropy & Minimum Norm & Capon & Beamforming \\
\hline Estimated Height $(\mathrm{m})$ & 120 & 16 & 66 & 48 & 141 \\
Difference $(\mathrm{m})$ & 0 & 104 & 54 & 72 & -21 \\
\hline
\end{tabular}




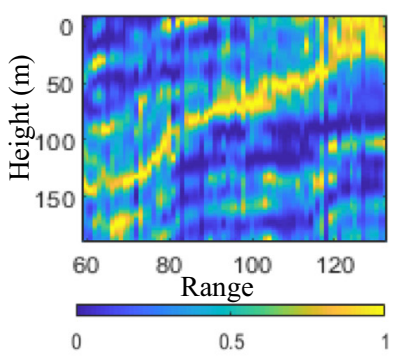

(a)

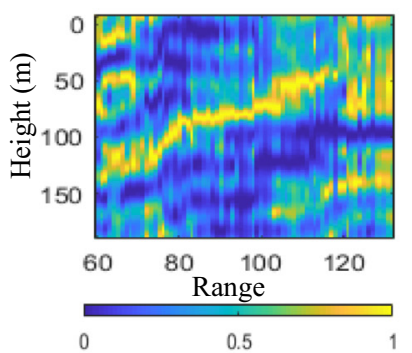

(b)

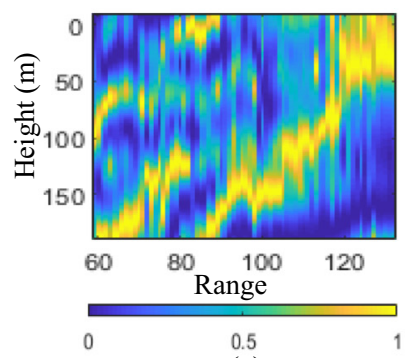

(c)

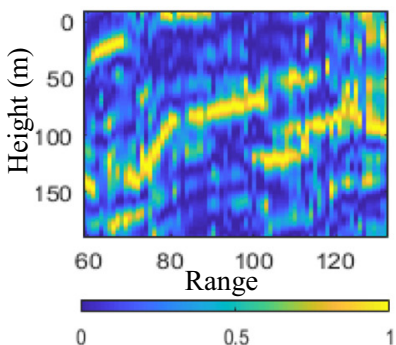

(d)

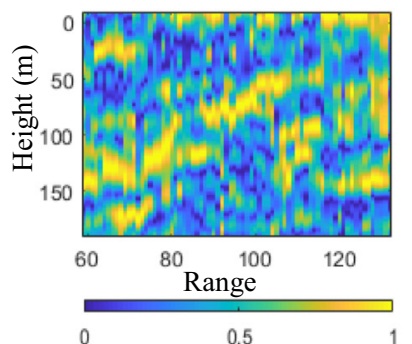

(e)

Fig. 5. Results of DOA estimation techniques over the Third Millennium Tower case study. (a) Linear Prediction. (b) Maximum Entropy. (c) Minimum Norm. (d) Capon. (e) Beamforming.

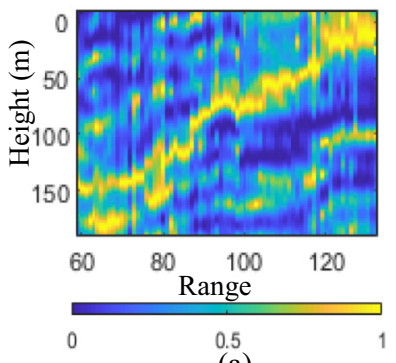

(a)

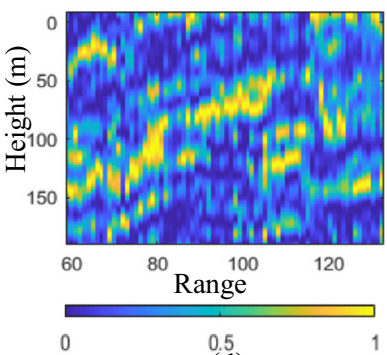

(d)

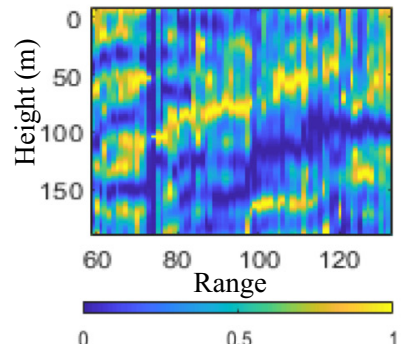

(b)

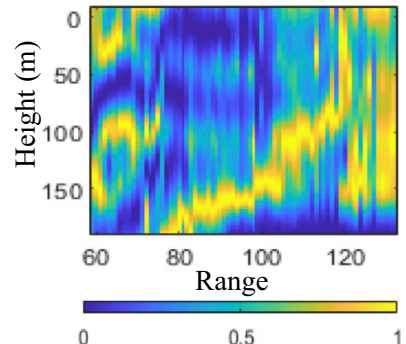

(c)

Fig. 6. Results of DOA estimation techniques over the Third Millennium Tower case study using 10 SAR images. (a) Linear Prediction. (b) Maximum Entropy. (c) Minimum Norm. (d) Capon. (e) Beamforming.

$N \times L$ matrix is initially created via by employing different columns of the identity matrix and the inverse of the covariance matrix, respectively. Then, for each pixel, the optimal vertical reflectivity profile vector is determined based on the contrast maximization. The contrast criterion can be described as Eq. (13):

$C\{\gamma(s)\}=\frac{\sqrt{\frac{1}{L} \sum_{m=1}^{L}\left(\gamma\left(s_{m}\right)-\frac{1}{L} \sum_{l=1}^{L} \gamma\left(s_{l}\right)^{2}\right)}}{\frac{1}{L} \sum_{m=1}^{L} \gamma\left(s_{m}\right)}$
Figs. 7 and 8 illustrate the results of optimal and random column selection from the identity matrix and the inverse of the covariance matrix, respectively, for both proposed LP and ME DOA estimation methods. The results show that the proposed approach can effectively select the best columns for the two SE techniques. Compared with the randomly chosen columns, the optimal column selection procedure allows obtaining accurate height-reflectivity profile reconstruction. 
Table 3

Estimated building heights and their differences for third millennium tower using 10 sar images.

\begin{tabular}{|c|c|c|c|c|c|}
\hline DOA Method & Linear Prediction & Maximum Entropy & Minimum Norm & Capon & Beamforming \\
\hline Estimated Height (m) & 122 & 8 & 32 & 5 & 90 \\
\hline Difference $(\mathrm{m})$ & -2 & 112 & 88 & 115 & 30 \\
\hline
\end{tabular}

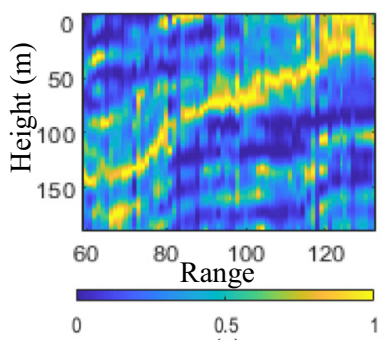

(a)

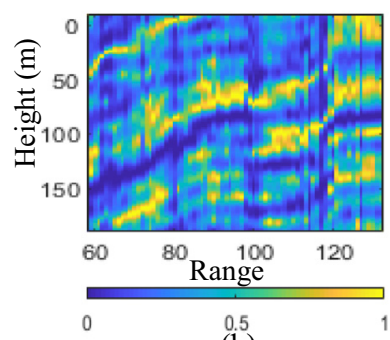

(b)
Fig. 7. Results of LP estimator for Third Millennium Tower. (a) Choosing the best column from the identity matrix. (b) Randomly select columns from identity matrix.

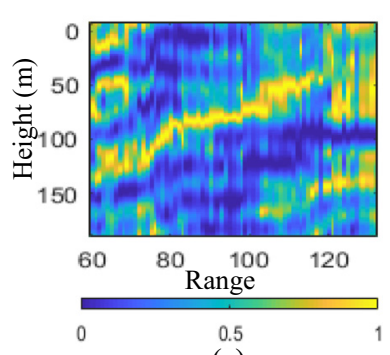

(a)

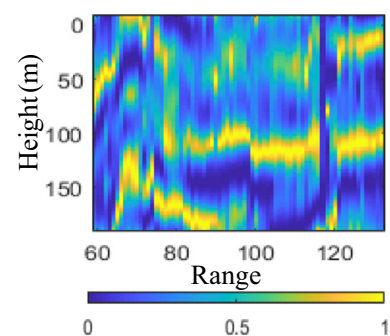

(b)
Fig. 8. Results of ME estimator for Third Millennium Tower. (a) Choosing the best column from the inverse of the covariance matrix. (b) Randomly select columns from inverse of the covariance matrix.

\section{Conclusion}

In this work, the performance of a novel non-parametric DOA estimation technique to improve the accuracy of building height estimates in urban areas is evaluated. The analysis of the proposed TomoSAR methods is based on the stack of 19 TerraSAR-X stripmap images from descending orbits in the city of Tehran, Iran. The Third Millennium Tower case study building is thus selected to analyze the efficiency of the DOA estimation methods. The obtained results present the capability of the LP estimator to resolve the layover effect in the urban environment by separating the single- and double-scattering mechanisms in the elevation direction. The proposed LP TomoSAR technique can enhance the reducing sidelobe interference in the reconstructed height-reflectivity profile. Compared with other employed SE methods, such as $\mathrm{ME}, \mathrm{MN}$, Capon, and $\mathrm{BF}$, the LP method provides a non-parametric DOA estimator for accurate estimation of the height of the buildings. As well, a robust estimate of building height is achieved while reducing the number of SAR of images.

\section{Declaration of Competing Interest}

The authors declare that they have no known competing financial interests or personal relationships that could have appeared to influence the work reported in this paper.

\section{Acknowledgement}

The authors would like to thank the Iran National Science Foundation (INSF) for generous financial support. The authors also thank the European Space Agency (ESA) for providing the TerraSAR-X data images.

\section{References}

Ermolaev, V.T., Gershman, A.B., 1994. Fast algorithm for minimumnorm direction-of-arrival estimation. IEEE Trans. Signal Process. 42 (9), 2389-2394.

Fornaro, G., 2014. Tomographic SAR. NATO SET-191 Lecture Series. Institute for Electromagnetic Sensing of the Environment (IREA), Italy, pp. 1-16.

Fornaro, G., Serafino, F., Soldovieri, F., 2003. Three-dimensional focusing with multipass SAR data. IEEE Trans. Geosci. Remote Sens. 41 (3), 507-517.

Gamba, J., 2020. Radar Signal Processing for Autonomous Driving, 1st ed. Singapore, Springer.

Gini, F., Lombardini, F., 2005. Multibaseline cross-track SAR interferometry: A signal processing perspective. IEEE Aerosp. Electron. Syst. Mag. 20 (8), 71-93.

Gini, F., Lombardini, F., Montanari, M., 2002. Layover solution in multibaseline SAR interferometry. IEEE Trans. Aerosp. Electron. Syst. 38 (4), 1344-1356.

Guillaso, S., D'Hondt, O., Hellwich, O., 2014. Urban scene reconstruction from a reduced number of tomographic SAR data. In: IEEE Geoscience and Remote Sensing Symposium (IGARSS), pp. 3164 3167.

Johnson, D.H., 1982. The application of spectral estimation methods to bearing estimation problems. Proc. IEEE 70 (9), 1018-1028.

Kato, T., Yamada, H., Yamaguchi, Y., Sato, R., Kojima, S., Arii, M., 2015. Fundamental study on multi-baseline SAR tomography using airborne X-band SAR. In: IEEE Asia-Pacific Conference on Synthetic Aperture Radar (APSAR), pp. 15-19.

Lombardini, F., Cai, F., Pasculli, D., 2013. Spaceborne 3-D SAR tomography for analyzing garbled urban scenarios: Single-look superresolution advances and experiments. IEEE J. Sel Top Appl Earth Obs Remote Sens 6 (2), 960-968.

Lombardini, F., Reigber, A., 2003. Adaptive spectral estimation for multibaseline SAR tomography with airborne L-band data. In: IEEE Geoscience and Remote Sensing Symposium (IGARSS), pp. 2014 2016.

Makhoul, J., 1975. Linear prediction: A tutorial review. Proc. IEEE 63 (4), $561-580$.

Martin Del Campo, G.D., Shkvarko, Y.V., Reigber, A., Nannini, M., 2018. TomoSAR imaging for the study of forested areas: A virtual adaptive Beamforming approach. Remote Sens. 10, 1-24.

Schmitt, M., Stilla, U., 2014. Comparison of multi-aspect multi-baseline SAR interferometry and multi-aspect TomoSAR reconstruction 
results. In: European Conference on Synthetic Aperture Radar, pp. 14.

Stilla, U., Soergel, U., Thoennessen, U., 2003. Potential and limits of InSAR data for building reconstruction in built-up areas. ISPRS J. Photogramm. Remote Sens. 58 (1-2), 113-123.

Stoica, P., Moses, R.L., 1997. Introduction to spectral analysis, first ed. New Jersey, Prentice Hall.

Stoica, P., Moses, R.L., 2005. Spectral analysis of signals. Prentice Hall, New Jersey.
Wei, L., Balz, T., Liao, M., Zhang, L.u., 2014. TerraSAR-X stripMap data interpretation of complex urban scenarios with 3D SAR tomography. J. Sens 2014, 1-7.

Zhu, X.X., 2011. Very high resolution tomographic SAR inversion for urban infrastructure monitoring-A sparse and nonlinear tour. Technische Universität München.

Zhu, X.X., Bamler, R., 2010. Tomographic SAR inversion by L1 norm regularization-The compressive sensing approach. IEEE Trans. Geosci. Remote Sens. 48, 3839-3846. 